Original article

\title{
DETECTION OF LISTERIA PATHOGENS BY GRADIENT/ CONSTANT DENATURING GEL ELECTROPHORESIS
}

\author{
A. AL-MARIRI, L. RAMADAN \& L. AL-HALAB \\ Department of Molecular Biology and Biotechnology, Atomic Energy \\ Commission, P.O. Box 6091, Damascus, Syria
}

\section{Summary}

Al-Mariri, A., L. Ramadan \& L. Al-Halab, 2018. Detection of Listeria pathogens by gradient/constant denaturing gel electrophoresis. Bulg. J. Vet. Med., 21, No 3, 322-335.

Listeriosis is a serious, but preventable disease, and the virulence factor of this disease is produced only by the two pathogenic species $L$. monocytogenes and $L$. ivanovii in humans and/or animals. In this study, we used both denaturing gradient gel electrophoresis (DGGE) and constant denaturing gel electrophoresis (CDGE) as molecular methods combined with Polymerase Chain Reaction (PCR) for the detection and identification of Listeria pathogens on 543 samples of raw milk collected from all Syrian provinces. The two methods are based on the PCR amplification of a fragment of the InlC gene (virulence gene) from the studied Listeria species and on the analysis of the PCR products obtained by DGGE/CDGE. Based on the differences present in the sequences amplified, it was possible to obtain species-specific DGGE/CDGE migration that allowed fast and easy identification of the virulence and pathogenicity of $L$. monocytogenes and $L$. ivanovii in humans and/or animals, in order to reduce the incidence of Listeria bacteria in the environment and foods and to prevent the occurrence of listeriosis in animal and human hosts.

Key words: constant denaturing gel electrophoresis (CDGE), denaturing gradient gel electrophoresis (DGGE), listeriosis, polymerase chain reaction (PCR), raw milk, virulence

\section{INTRODUCTION}

Listeriosis is a dangerous disease. Listeria spp. are ubiquitous Gram-positive bacteria present in a diversity of environments, including soil, vegetation, and water, where they survive as saprophytes (AlMariri et al., 2013). Extensive research efforts in the preceding decades led to determination of two Listeria pathogens. L. monocytogenes was first described in the 1920s, but its impact as a food-borne pathogen was not fully appreciated until the 1980s (McLauchlin, 1997), although it is apparent that $L$. monocytogenes causes an extremely serious, invasive, and often life-threatening food-borne disease (listeriosis). In very rare instances, this disease is due to Listeria ivanovii (previously known as L. monocytogenes serotype 5) (Guillet et al., 2010). Although some early clinical manifestations of human listerio- 
sis are mild, nonspecific, and influenzalike (e.g., chills, fatigue, headache, muscular and joint pain, as well as gastroenteritis), serious systemic illness occurs, including septicaemia, meningitis, encephalitis, abortions, and, in some cases, death (Liu et al., 2012).

The average mortality rate from human listeriosis around the world is $30 \%$, which is higher than that for most of other common food-borne pathogens such as Salmonella enteritidis (with a mortality of $0.38 \%)$, Campylobacter species $(0.02-$ $0.1 \%)$, and Vibrio species (0.005-0.01\%), (Mead et al., 1999).

The early generation laboratory diagnostic tests for L. monocytogenes are largely phenotype-based and usually assess L. monocytogenes gene products by biological, biochemical, and serological means. As the phenotypic properties of Listeria may vary with the constantly changing external conditions, growth phase, and spontaneous genetic alterations, use of the phenotype-based diagnostic procedures may lead to equivocal results at times. In addition, since many phenotypic tests are dependent on lengthy in vitro culture procedures, they are notoriously time consuming, thus delaying result availability. To circumvent the drawbacks of the phenotype-based procedures, new generation genotype-based methods targeting the nucleic acids (DNA or RNA) of Listeria have been developed over the preceding decade. As nucleic acids (particularly DNA) are intrinsically more stable than proteins and less prone to influences by external factors, the genotypic (or molecular) diagnostic procedures are much more precise and less variable than the phenotype-based methods. Furthermore, as many of the genotypic techniques involve template and/or signal amplification, they are also much faster and more sensitive. Application of these stateof-the-art genotypic identification procedures such as denaturing gradient gel electrophoresis (DGGE) and constant denaturing gel electrophoresis (CDGE) attaching with PCR technology have the potential to overcome the problems associated with the phenotype-based tests, providing unprecedented levels of sensitivity, specificity, and speed for laboratory detection and identification of Listeria without the need to culture the suspect microorganism(s) (Cocolin et al., 2002).

Denaturing gradient gel electrophoresis (DGGE) is one of several methods that can be used to screen DNA fragments for small sequence changes or point mutations. The separation principle of DGGE is based on the melting behaviour of DNA molecules, whereas CDGE is a modification of denaturing gradient gel electrophoresis.

After a mutation has been identified by previous DGGE gels, a CDGE gel can be used to rapidly screen samples for the presence of a mutation. Then, DGGE, which is based on the discontinuous phenomenon of strand dissociation, allows the resolution of DNA fragments differing by as little as a single nucleotide substitution. In mutation analysis using parallel DGGE, field strength, temperature, and run time must be strictly controlled to achieve reproducible results.

DGGE is complicated by the difficulties of choosing the exact running time and gel conditions to achieve the optimal separation. By running a DGGE gel too long, an achieved separation decreases, and may even be lost. Mutations present in a homo- or hemizygote state, where heteroduplexes are not formed, will be missed. Several of these problems are eliminated by CDGE in which a single denaturing condition is used to melt a 
fragment. In CDGE, wild-type and mutant fragments will melt partially into a certain configuration immediately after entering the gel. This configuration will be kept throughout the run, and the fragments will migrate with a constant rate. Therefore, the longer the gel is run, the wider the separation between the mutant and wildtype samples.

DGGE/CDGE are useful techniques to monitor microbial populations, without culture dependant methods (Adil, 2015). PCR-DGGE is sorted as part of the new discipline of microbial population as Pyrenophora and Mycoplasma species (Mcauliffe et al., 2005; Mavragani et al., 2011).

The combined DGGE/CDGE system was chosen as it might have the advantages of both systems; heteroduplex molecules may become visible in the DGGE component, while the CDGE component should prevent complete strand dissociation.

In our study, specific primers for listerial pathogens were targeted typically to a sequence of a gene coding for a virulence factor (Internalin "InlC" ) unique to L. monocytogenes, L. ivanovii (Engelbrecht et al., 1998; Vazquez-B et al., 2001) in order to highlight the ability of PCR-DGGE/CDGE for detection of Listeria in milk (Quigley et al., 2013). Then the PCR products have been analysed by density gradient gel electrophoresis (DGGE) (perpendicular or parallel denaturing gel) which was able to separate DNA fragments of the same size when the fragments display differences in the sequence of the base pairs that yield different $\mathrm{G}+\mathrm{C}$ content, while these fragments have been resolved as discrete bands on a gel, so the denaturant concentration that gives optimal resolution from a parallel or perpendicular DGGE gel is held constant. This means that the optimum concentra- tion of denaturant to use for a CDGE is determined from the visible maximum split on the gel which distinguish between two Listeria pathogens.

In this study, we aimed to show the capability of DGGE/CDGE with respect to their use as a fast screening test to investigate the presence of Listeria pathogens in the studied samples.

\section{MATERIALS AND METHODS}

\section{Bacterial strains}

Listeria spp. strains in this study were isolated from 543 raw milk samples (370 bovines, 173 sheep) which were collected from different provinces of Syria (collected from unorganised sectors) from September 2010 to December 2013. Then, these strains were cultured on brain heart infusion (BHI; Oxoid) broth and incubated at 30 or $37^{\circ} \mathrm{C}$ overnight before being subjected to analysis by molecular methods.

\section{Traditional isolation of Listeria pathogens}

Previous isolations were identified by using traditional methods. In particular, Gram staining, catalase test, oxidase activity, $\mathrm{H}_{2} \mathrm{~S}$ production, mobility test, $\beta$-haemolysis, camp test, and production of acids from rhamnose and xylose were used (Iordache \& Tofan, 2008; Al-Mariri et al., 2013).

\section{DNA extraction}

Briefly, for the extraction of Listeria DNA, $1 \mathrm{~mL}$ of pure Listeria culture was centrifuged at $13.000 \times \mathrm{g}$ for $5 \mathrm{~min}$ at room temperature. The DNA was then extracted using a genomic DNA purification kit (Fermentas, GmbH, Germany), and the DNA concentration was determined using 
a spectrophotometer (NanoDrop 2000, Thermo Fisher Scientific mbH, Germany) at $260 \mathrm{~nm}$.

\section{PCR amplification of target regions}

The PCR primers used for the amplification of DNA fragments spanning the Inlc gene (member of Internalin multigene family) for L. monocytogenes and L. ivanovii were targeted at highly conserved regions of this gene. Primers for the detection of the Listeria spp. isolates are given in Table 1.

Five hundred ng of extracted genomic DNA were used for PCR amplification. The PCR reaction mixture $(25 \mu \mathrm{L})$ contained $5 \mu \mathrm{L}$ PCR buffer HotStar HiFidelity (Qiagen, Germany), $200 \mu \mathrm{M}$ of each dNTP (Promega, USA), $2.5 \mathrm{mM} \mathrm{MgCl}_{2}$, 25 pmol of each primer and 1.5 U HotStar HiFidelity DNA Polymerase (Qiagen, Germany) according to standard protocols (Innis et al., 1990; Luo et al., 2004).

PCR amplification reactions were performed in a GeneAmp ${ }^{\circledR}$ PCR system 9700 (Applied Biosystems, USA), (30-40 pb) GC clamps were added to one of pair primers (5'- GCG GGC GGC GCG GGG
CGC GGG CAG GGC GGC GGG GGC GGG GG C GGC -3') to improve its sensitivity in the detection of DNA sequence by DGGE analysis as previously described. To minimise non-specific annealing of the primers, Taq polymerase was added after the first denaturing step, at a temperature of $80^{\circ} \mathrm{C}$, a technique referred to as "Hot Start". Another technique (Muyzer et al., 1995) referred to as "Touchdown", was also used to reduce the formation of spurious by-products (Wawer \& Muyzer, 1995). This involved setting the annealing temperature (Tan) $10{ }^{\circ} \mathrm{C}$ higher than the calculated primer melting temperature for the first cycle, then decreasing it by $0.5{ }^{\circ} \mathrm{C}$ every cycle for 20 cycles, and finally annealing at the Tm during 9 subsequent cycles as shown in Table 2.

The data in the table explain that when the reaction of PCR was carried out with primer pair mon/ivaF (with GC clamp) and mon/ivaR, the annealing temperature (Touchdown) was set at $66^{\circ} \mathrm{C}$ for the first cycle and decreased by $0.5^{\circ} \mathrm{C}$ each cycle thereafter for 20 cycles, with the final 9 cycles performed at $\mathrm{Tm}$ of $56^{\circ} \mathrm{C}$ as shown.

Table 1. Primers used for PCR and amplification of InlC region of Listeria spp. isolates

\begin{tabular}{ll}
\hline Primer sequence (5'-3') & \multicolumn{1}{c}{ Listeria spp. } \\
\hline GCGGGCGGCGCGGGGCGCGGGCAGGGCGGCGGGGGCGGGC & L. monocytogenes \\
CTCTTTTGTGTTTCTAAATTTCATTTTGTAACCAATTATTTT & L. ivanovii \\
\hline
\end{tabular}

Table 2. Hot Start and Touchdown PCR protocol

\begin{tabular}{lll}
\hline $94^{\circ} \mathrm{C}$ & $3 \mathrm{~min}$ & \\
$80^{\circ} \mathrm{C}$ & $1 \mathrm{~min}$ & \\
\hline $66^{\circ} \mathrm{C}$ & $1 \mathrm{~min}$ & Repeated 20 times with the annealing temperature decreased by $0.5^{\circ} \mathrm{C}$ \\
$75^{\circ} \mathrm{C}$ & $45 \mathrm{sec}$ & every cycle \\
\hline $56^{\circ} \mathrm{C}$ & $1 \mathrm{~min}$ & \\
$75^{\circ} \mathrm{C}$ & $45 \mathrm{sec}$ & Repeated 9 times at $\mathrm{Tm}$ \\
$94^{\circ} \mathrm{C}$ & $1 \mathrm{~min}$ & \\
\hline $61^{\circ} \mathrm{C}$ & $45 \mathrm{sec}$ & \\
$75^{\circ} \mathrm{C}$ & $10 \mathrm{~min}$ & \\
\hline
\end{tabular}


In the final step of the reaction, the mixture was held at $75^{\circ} \mathrm{C}$ for 10 min to allow the extension of incomplete products (Nübel et al., 1996; Aznar et al., 2002).

The amplified products were analysed by agarose gel electrophoresis as follows. Ethidium bromide (Sigma, Germany) was added to $2 \%$ agarose solution at a concentration of $0.5 \mathrm{mg} / \mathrm{mL}$ before the gel was poured in the mold. The gel was run for $1 \mathrm{~h}$ at $50 \mathrm{~V}$, and the PCR product was visualised under UV-light. Then, the PCR product was purified using a QiaQuick PCR purification kit (Qiagen, Milan, Italy) and sequenced by a commercial facility (MWG Biotech, Edelsberg, Germany). The amplified products were stored at $-20{ }^{\circ} \mathrm{C}$ until they were used for DGGE.

\section{$D G G E$ analysis}

The Dcode Universal Mutation Detection system (Bio-Rad, Hercules, USA) was employed to separate the sequencespecific of the PCR products.

\section{Perpendicular gradient gel analysis}

This analysis uses a range of denaturants to separate the two Listeria pathogens DNA fragments. Electrophoresis was performed in a $1 \mathrm{~mm}$-thick $(16 \times 16 \mathrm{~cm})$ polyacrylamide gel $(10 \% \mathrm{w} / \mathrm{v}$ acrylamidebisacrylamide $37.5: 1$ ) containing 20 to $70 \%$ urea/formamide denaturing gradient ( $100 \%$ denaturant is a mixture of $7 \mathrm{M}$ urea and $40 \% \mathrm{v} / \mathrm{v}$ formamide) (McAuliffe et al., 2005), increasing in the direction of the electrophoretic run. The gel was cast using a Bio-Rad Model 385 Gradient Delivery System (Bio-Rad Laboratories, Hercules, CA).

We added $100 \mu \mathrm{L} 2 \times$ gel loading dye (70\% glycerol, $0.05 \%$ bromophenol blue, $0.05 \%$ xylene cyanole, $2 \mathrm{mM}$ EDTA) to the $100 \mu \mathrm{L}$ equal mixer of the samples (amplified DNA of L. monocytogenes and
L. ivanovii), and electrophoresed them on the Dcode universal mutation detection system at $130 \mathrm{~V}$ for $2 \mathrm{~h}$ at $65^{\circ} \mathrm{C}$. After electrophoresis, the gels were stained in a $50 \mu \mathrm{g} / \mathrm{mL}$ ethidium bromide in $1 \times \mathrm{TAE}$ buffer for $3 \mathrm{~min}$, then distained the gel in $1 \times$ TAE buffer for about $20 \mathrm{~min}$, The gels were imaged under ultraviolet transillumination.

\section{Parallel gradient gel analysis}

For this analysis, the denaturant is parallel to the electrophoresis direction. A time course run is initially done for parallel gels to find the optimal run time for the $L$. monocytogenes and L. ivanovii DNA samples. Here, electrophoresis was performed in a $1 \mathrm{~mm}$-thick $(16 \times 16 \mathrm{~cm})$ polyacrylamide gel $(10 \% \quad \mathrm{w} / \mathrm{v}$ acrylamidebisacrylamide $37.5: 1$ ) containing $40-65 \%$ urea/formamide denaturing gradient. Five $\mu \mathrm{L}$ of PCR products were mixed with 5 $\mu \mathrm{L} 2 \times$ gel loading dye, and electrophoresed on the Dcode universal system at 150 $\mathrm{V}$ for $2.5 \mathrm{~h}$ at $65{ }^{\circ} \mathrm{C}$. After electrophoresis, the gels were stained in a $50 \mu \mathrm{g} / \mathrm{mL}$ ethidium bromide in $1 \times$ TAE buffer for 3 min, then destained in $1 \times$ TAE buffer for about $20 \mathrm{~min}$, and imaged under ultraviolet transillumination (Abu Al-Soud et al., 2003).

\section{Constant gradient gel analysis}

Constant denaturing gel electrophoresis is a modification of DGGE. In CDGE, the denaturant concentration that gives optimum resolution from a parallel or perpendicular DGGE gel is held constant. For this analysis, the optimal concentration of denaturant is determined from the maximum split between L. monocytogenes and L. ivanovii DNA, as seen in the perpendicular or parallel denaturing gel. Here, electrophoresis was performed also in a $1 \mathrm{~mm}$-thick $(16 \times 16 \mathrm{~cm})$ polyacrylamide 
gel $(10 \% \quad \mathrm{w} / \mathrm{v}$ acrylamide-bisacrylamide 37.5:1) containing a constant urea/formamide denaturing 51\% gradient, $180-300$ ng of amplified DNA was mixed with an equal volume of $2 \times$ gel loading dye, and electrophoresed on the Dcode universal system at $130 \mathrm{~V}$ for $2.5 \mathrm{~h}$ at $65^{\circ} \mathrm{C}$. Then, after electrophoresis, the protocol was as mentioned in both previous analyses $(\mathrm{Wu}$ et al., 1999; Farnleitner et al., 2000).

\section{Heteroduplexes preparation for DGGE analysis}

It is important to optimise the PCR reaction to minimise unwanted products which may interfere with gel analysis. Heteroduplexes could be generated during PCR by amplifying DNA of L. monocytogenes and L. ivanovii samples in the same tube. The tube was heated at $95^{\circ} \mathrm{C}$ for $5 \mathrm{~min}$, then placed at $65{ }^{\circ} \mathrm{C}$ for $1 \mathrm{~h}$, and let slowly to cool to room temperature (Tchernitchko et al., 1999).

\section{RESULTS}

\section{Isolation of Listeria}

Among the 543 samples, thirty-nine Listeria strains were identified: 29 as $L$. monocytogenes (43\%), and 10 - as L. ivanovii $(15 \%)$. All isolates were Gram positive bacteria, regular, short rods, 0.4-0.5 by $1-2 \mu \mathrm{m}$ of size, with parallel sides, and blunt ends and usually occured singly or in short chains in Oxford and Palcam agar cultures when incubated at $30^{\circ} \mathrm{C}$ for $48 \mathrm{~h}$, catalase-positive and oxidase-negative.

Results of amplification InlC gene as a target for specific Listeria pathogens

The set of primers was designed based on the partial alignments of the $I n l C$ gene from the Listeria pathogens (L. monocytogenes, L. ivanovii), the primers chosen were able to prime the amplification of the two pathogens tested, due to their high or complete homology to the sequence of the InlC gene considered. More specifically, two primers (forward, reverse) were completely homologous to the reference sequence of the two Listeria species under consideration. So, these primers were able to amplify a region that contained only one significant difference (G: in L. monocytogenes - 5'CTCTTTT GTGTTTCTAA ATTTATTTTTAAGGAGTGGAGAATG TTGAAAAAAAATAATTGGTTACAA

AATG3'; A: in L. ivanovii: 5'CTCTTT TGTGTTTCTAAATTTATTTTTAAGG AGTGGAGAATATTTGAAAAAAAATA ATTGGTTACAĀAATG3').

Primer pair (mono, iva)F/R yielded a single band on agarose gel corresponding to a 70 bp DNA fragment when the PCR reaction was carried out with Taq polymerase on DNA extracted from tested $L$. monocytogenes, L. ivanovii samples. That a single band was obtained with each primer pair indicated that only the target gene was amplified, with no non-specific primer attachment or heteroduplexes formed as seen in Fig. 1, which summarises the amplification results by PCR reaction of the target DNA of InlC gene of two Listeria strains using specific primers sets: lanes 1, 4 indicated standard L. monocytogenes gene and L. ivanovii, respectively (70 bp); lanes 2, 3 - tested L. monocytogenes, L. ivanovii, respectively (also 70 bp), whereas lane 5 - genus Brucella which in our lab was used as negative control.

All isolates were well-defined as $L$. monocytogenes, L. ivanovii by PCR reaction, in order to study them by DGGE analysis.

\section{Perpendicular gradient gel analysis}

In perpendicular denaturing gradient gel, a range of denaturants $(20-70 \%)$ was used 
Detection of Listeria pathogens by gradient/constant denaturing gel electrophoresis

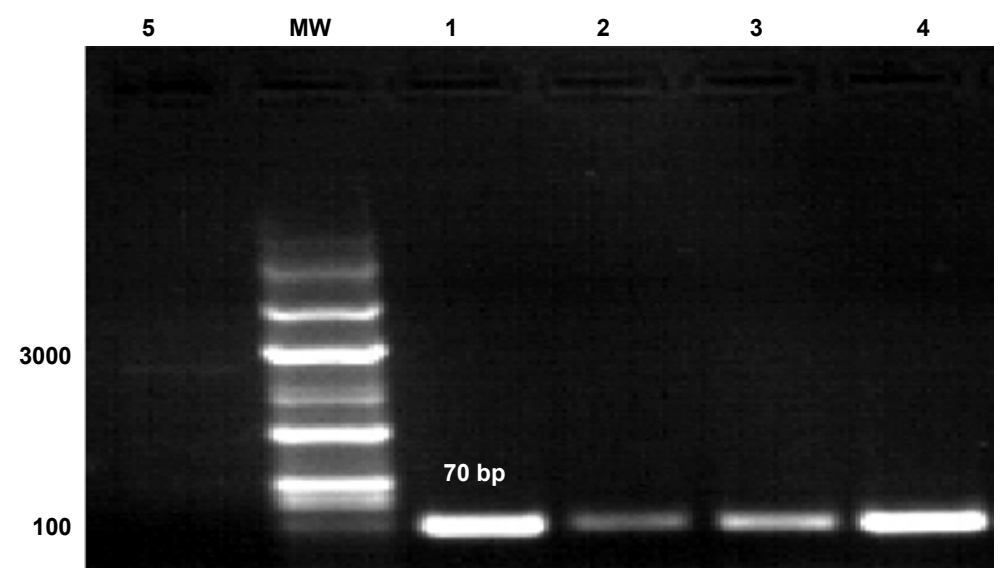

Fig. 1. Electrophoresis on $1.5 \%$ agarose gel showing the PCR products of InlC gene. MW: DNA ladder; lane 1: amplification of standard L. monocytogenes; lane 2: amplification of tested L. monocytogenes; lane 3: amplification of tested L. ivanovii; lane 4: amplification of standard L. ivanovii, lane 5: refered to genus Brucella which was classified in our lab as negative control.

to separate the L. monocytogenes and $L$. ivanovii strain DNA fragments of InlC gene. Both were electrophoresed on a perpendicular denaturing gradient gel, and the DNA pattern formed a "S" shape. A spilt (difference in migration) was formed between the L. monocytogenes and L. ivanovii strain DNA. This separation was caused by the L. ivanovii DNA melting sooner than the L. monocytogenes DNA of the InlC fragment as shown on Fig. 2. On this figure, the perpendicular gels had an increasing gradient of denaturants from left to right, perpendicular to the direction of electrophoresis. During electrophoresis, the migrating double-stranded fragment (arrow three) began to melt upon exposure of various domains of the fragment to an increasing denaturant concentration. Domains with a low $\mathrm{G}+\mathrm{C} \%$ (as L. ivanovii DNA, arrow one) melted at a low denaturant concentration, while domains with high $\mathrm{G}+\mathrm{C} \%$ (as L. monocytogenes DNA, arrow two) melted at higher denaturant concentrations further down the gel.

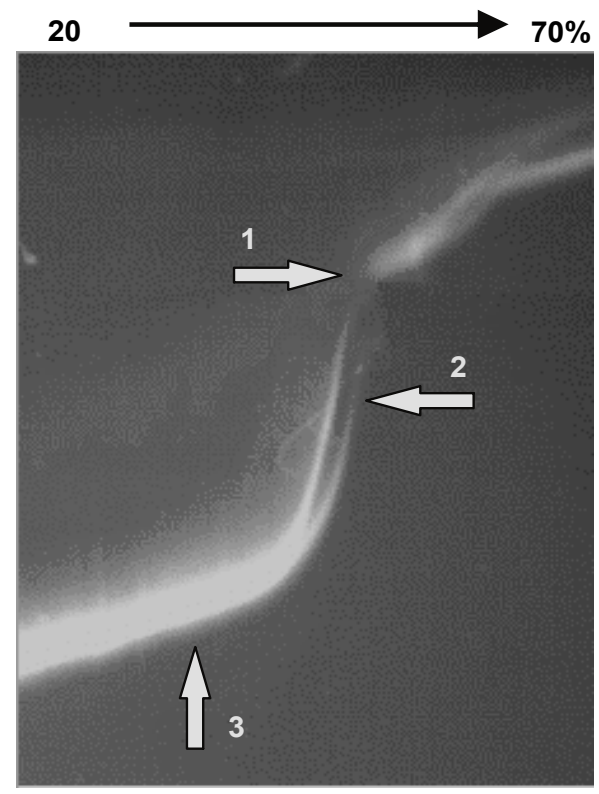

Fig. 2. Separation of $L$. monocytogenes and $L$. ivanovii-strain DNA electrophoresed on a 20 $70 \%$ perpendicular denaturing gradient gel. The gel was $10 \%$ acrylamide:bis $(37: 5: 1)$, run at $130 \mathrm{~V}$ in $1 \times \mathrm{TAE}$ buffer, heated at $65^{\circ} \mathrm{C}$ for $2 \mathrm{~h}$ (arrow 1: DNA L. ivanovii, arrow 2: DNA L. monocytogenes, arrow 3: DNA double stranded of both DNA of L. monocytogenes and L. ivanovii). 


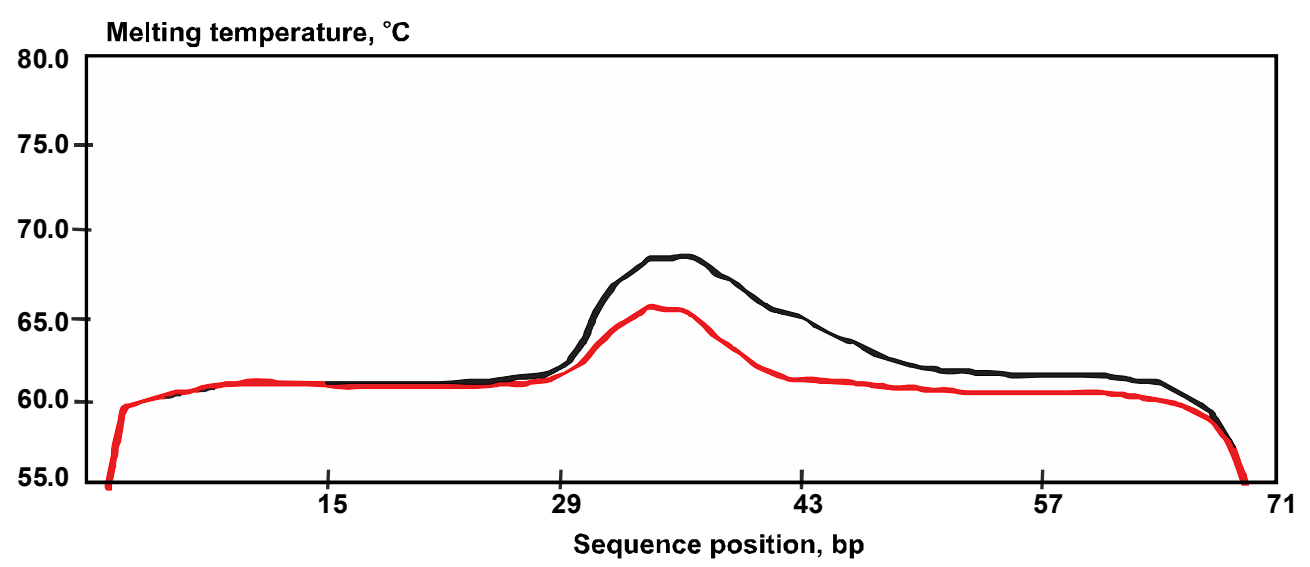

Fig. 3. The melting profile for the Internalin $(I n l C)$ sequence, it shows the spilt between the two sequences of $L$. monocytogenes (upper line) and L. ivanovii (lower line) DNA to determine the single base substitution ( $\mathrm{G}$ to $\mathrm{A}$ ), the melting profile for the Internalin sequence graphed using the $50 \%$ melted probability.

Therefore, the behaviour of a DNA fragment in a denaturing gradient gel could be predicted solely from the basepair sequence. On the other hand, a computer program calculated the $\mathrm{Tm}$ for each base pair, and the result determined a calculated melting map (Fig. 3), which showed high- and low-temperature melting domains. Only single base substitution was detected ( $\mathrm{G}$ in L. monocytogenes, A in L. ivanovii) in the low temperature melting domain of Internalin molecule as seen on Fig. 3, the spilt between the two sequences of L. monocytogenes and L. ivanovii DNA to determine the single base substitution ( $G$ to $A)$, the melting profile for the Internalin sequence graphed using the $50 \%$ melted probability. The $50 \%$ melting probability is typically used for analysing melting profiles for denaturing gel electrophoresis. From the melting profile, it can be seen that there were two low-temperature melting regions and one high-temperature melting region in the middle of the sequence.

\section{Parallel gradient gel analysis}

In parallel DGGE, the denaturing gradient was parallel to the electric field, and the range of denaturant was narrowed to allow better separation of fragments. In this analysis, we used a range of denaturants (40$65 \%$ ) to separate the L. monocytogenes and L. ivanovii-strain DNA fragments of internalin gene as shown on Fig. 4.

The parallel gels had an increasing gradient of denaturants from top to bottom, parallel to the direction of electrophoresis. Alignment of the base sequences of the InlC fragments targeted by the primer pair (mon, ivaF/R) had revealed one base that differed in the two fragments ( $\mathrm{G}$ in L. monocytogenes, $\mathrm{A}$ in $L$. ivanovii), so this difference yielded a DNA fragment of $L$. ivanovii with lower $\mathrm{G}+\mathrm{C} \%$, melting at a lower denaturant concentration to produce a DGGE band that migrated a shorter distance through the gel (lane 1) than the band produced from L. monocytogenes fragment (lane 2), 
while lane 3 contained the two Listeria pathogens fragments.
1

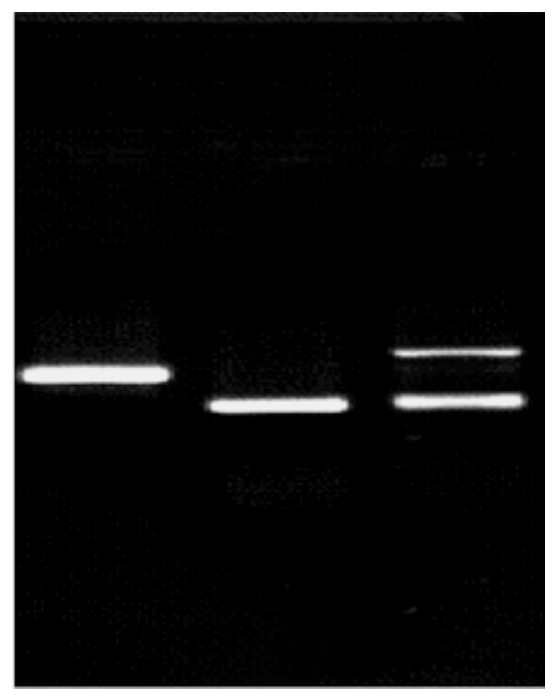

Fig. 4. Separation of $L$. monocytogenes and $L$. ivanovii strain DNA electrophoresed on a 40 $65 \%$ parellel denaturing gradient gel. The gel was $10 \%$ acrylamide:bis $(37: 5: 1)$, run at $150 \mathrm{~V}$ in $1 \times \mathrm{TAE}$ buffer, heated at $65^{\circ} \mathrm{C}$ for $2.5 \mathrm{~h}$. Lane 1: DNA of L.ivanovii; lane 2: DNA of L.monocytogenes; lane 3: DNA of two Listeria pathogens.

\section{Constant gradient gel analysis}

The L. monocytogenes and L. ivanovii amplified DNA run on a constant denaturing gel (CDGE). For this gel, a single denaturant concentration was used to melt the fragments of Inlc gene. The concentration of denaturant for CDGE was determined at the maximum split between the two studied Listeria species DNA, as seen in a perpendicular denaturing gradient gel.

The concentration of denaturant for CDGE gel was calculated first by placing a fluorescent ruler along the axis of the denaturant gradient when taking a photograph as seen in Fig. 5 which explained that the distance along the gradient where the maximum spilt seen between two Listeria strains was $5 \mathrm{~cm}$. This distance was divided by the length of the gel and multiplied by the denaturant range (5-8) $\times$ $50 \%=31 \%$. Then, this number was added to the starting denaturant concentration to determine the optimum concentration to use for CDGE $(20 \%+31 \%=51 \%)$. Therefore, the CDGE gel used a denaturant concentration of $51 \%$.

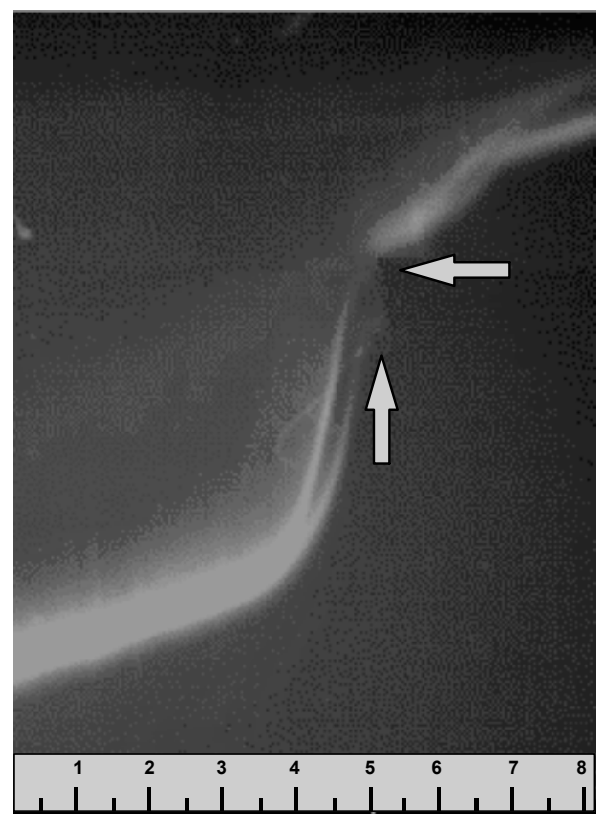

Fig. 5. Perpendicular DGGE gel used for determining the optimum denaturant concentration used in a CDGE gel.

\section{Heteroduplexes analysis}

Heteroduplex analysis (HA) was based on conformational differences in doublestranded DNA caused by the formation of heteroduplex molecules. Heteroduplex molecules had a mismatch in the doublestrand, caused a distortion in its usual conformation and detected on parallel gel due to slower migration than the correspon- 
ding homoduplex molecules. Heteroduplex molecule with as little as one mismatch could show a difference in mobility in a gel than homoduplex molecules as seen on Fig. 6 which showed that melting behaviour of the heteroduplexes was altered so that they melt at a lower denaturant concentration than the homoduplexes and could be visualised on a parallel denaturing gradient gel.

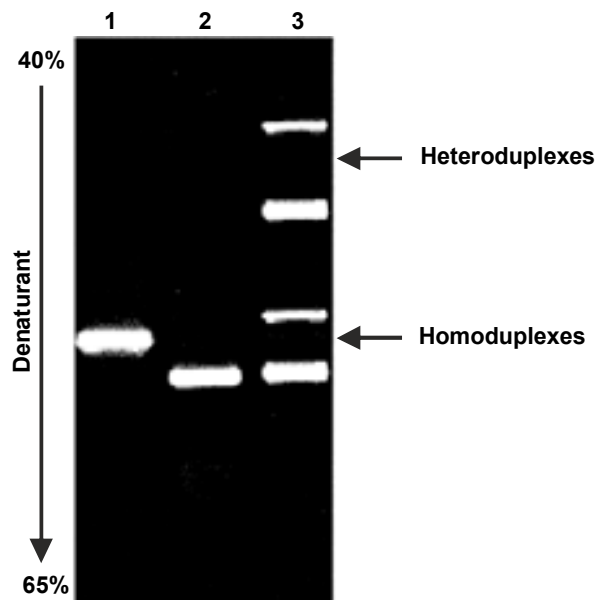

Fig. 6. L. monocytogenes and L. ivanovii DNA fragments that were denatured and re-annealed to generate four fragments run on a parallel denaturing gradient gel. Lane 1: L. ivanovii DNA, Lane 2: L. monocytogenes DNA, Lane 3: two heteroduplexes and two homoduplexes of L. monocytogenes and L. ivanovii.

\section{DISCUSSION}

Amplification of DNA by genus-specific primers that target DNA sequences and the subsequent differentiation of this DNA on DGGE gel is known as PCR-DGGE (Ercolini et al., 2004; Dar et al., 2005). It is a rapid and efficient separation technique of the same length DNA sequences (amplified by PCR), which may vary as little as a single base pair modification as in our study on Inlc gene for the determination of the two Listeria pathogens.

In this study, the development of a PCR-DGGE method to directly identify the pathogens of the genus Listeria ( $L$. monocytogenes and L. ivanovii) in milk samples is described. This approach exploited the potential of PCR to amplify, with specific primers, variable regions within the Inlc gene as well as the discriminatory power of DGGE/CDGE to differentiate DNA molecules on the basis of differences in their sequence (Luo et $a l ., 2004)$. The Inlc gene has been demonstrated to be a reliable PCR target for the differentiation of Listeria spp., (Eruteya \& Odunfa, 2014).

The fragment of studied Inlc gene was characterised from different Listerial pathogens and demonstrated the presence of conserved regions at the $5^{\prime} / 3^{\prime}$ ends and a species-specific internal region. This gene was used to design primers that specifically amplify Listeria spp., (Moreno et al., 2014) and that it could be used for specific identification using the DGGE/ CDGE method. Two primers were identified for the region of the sequences that were conserved among different Listeria spp. The regions amplified contained a high degree of heterogeneity among the sequences considered, allowing their differentiation by DGGE/CDGE. The PCR products produced from studied samples were used to optimise the experimental conditions (denaturant gradients, temperature, voltage, and length of the electrophoretic run) of the DGGE (perpendicularDGGE/parallel-DGGE and CDGE). Denaturants from 40 to $65 \%$ in the parallelDGGE showed the best differentiation power, allowing the identification, on the basis of specific migration, of the two pathogens considered in the study which agreed with the study of Luca Cocolin et 
al. (2002) about using DGGE in detecting the food-borne bacterial pathogen $L$. monocytogenes by the amplification the iap gene, while, the iap gene has been also demonstrated to be a reliable PCR target for differentiation of Listeria spp. (Rawool et al., 2007). Simultaneously, Petersen et al. (2007) explained that the PCR combined with DGGE as capable to distinguish another species of bacteria as Campylobacter, Helicobacter, Arcobacter and related Epsilobacteria in saliva samples from humans and domestic pets.

Furthermore, this methodology can be utilised in diverse subject areas. For instance, DGGE allowed the identification of over 65 Mycoplasma species of human and veterinary origins in less than 24 hours (McAuliffe et al., 2005). Mycoplasmas are fastidious organisms that require many weeks to culture and other serological tests to be identified. They cause various diseases associated with pneumonia, arthritis, conjunctivitis, infertility and abortion. Moreover, a total of 16 Bifidobacterium species were rapidly identified by PCR-DGGE method targeted on a 770-bp region of the tuf gene (Sheu et al., 2013). These applications of PCR-DGGE could potentially allow considerable savings of time, life and treatment costs.

Also, Dang et al. (2002) assured the importance of DGGE which is one of the most consistently used mutation scanning methods by using of single base changes in the $p 53$ gene to detect minimal residual disease of breast cancer. Mark McCammon et al. (2005) demonstrated the power of DGGE in exposing of pncA mutations associated with pyrazinamide resistance in Mycobacterium tuberculosis isolates from the United states-Mexico Border Region. In addition, DGGE has been established to have a real potential in screening a large number of patients for rapid and reliable identification of deleterious changes in the oncogenes BRCA1 and sBRCA2 (Van der Hout et al., 2006). Hence PCR-DGGE allowed the detection of numerous mutations and revealed the existence of unclassified variants that were not reported before. Over and above, the recent studies by Liao et al. (2015) aimed to simultaneously construct PCRDGGE-based predictive growth models of Listeria monocytogenes and Vibrio parahaemolyticus on cooked shrimps at 4 and $10^{\circ} \mathrm{C}$, but it is inappropriate for inactivation models yet.

It is to be noted that PCR-DGGE became more important when combined with CDGE, which we tested in our study when the optimum concentration of denaturant on the electrophoresis gel was determined. This matter was explained by Wu et al., (1999) whereas the combination between DGGE and CDGE had the advantages of both methods, because it prevented the dissociation of the total strand DNA, raised the capability of separation the heteroduplex molecules, and it was proved successful in the detection of DNA variants in several GC-rich fragments.

\section{CONCLUSIONS}

So, from all mentioned previous data, we concluded that using of DGGE/CDGE technique, diagnosis of emerging infections could become easier and faster, and identification of uncultivable pathogens can also now be facilitated, because, understanding complex microbial populations would certainly help us in rapid decision-making with regard to adequate treatment and other major interventions aiming at making the world a better and safer place to live. 


\section{ACKNOWLEDGEMENTS}

The authors would like to thank the General Director of the AECS and the Head of the Molecular Biology and Biotechnology Department for their support.

\section{REFERENCES}

Abu Al-Soud, W. M. Bennedsen, S. L. W. On, I. S. Ouis, P. Vandamme, H. O. Nilsson, A. Ljungh \& T. Wadstrom, 2003. Assessment of PCR-DGGE for the identification of diverse Helicobacter species, and application to faecal samples from zoo animals to determine Helicobacter prevalence. Journal of Medical Microbiology, 52, 765-771.

Adil, E., 2015. Corrective measures of denaturing gradient gel electrophoresis limitations. Journal of Environmental Science and Technology, 8, 1-12.

Al-Mariri, A., A. Abou Younes \& L. Ramadan, 2013. Prevalence of Listeria spp. in raw milk in Syria. Bulgarian Journal of Veterinary Medicine, 16, 112-122.

Aznar, R. \& B. Alarcon, 2002. On the specificity of PCR detection of Listeria monocytogenes in food: A comparison of published primers. Systematic \& Applied Microbiology, 25, 109-119.

Cocolin, L., K. Rantsiou, L. Iacumin, C. Cantoni \& G. Comi, 2002. Direct identification in food samples of Listeria spp. and Listeria monocytogenes by molecular methods. Applied \& Environmental Microbiology, 68, 6273-6282.

Dang, R. K. B., R. S. Anthony, J. I. O. Craig, R. C. F. Leonard \& A. C. Parker, 2002. Limitations of the use of single base changes in the $p 53$ gene to detect minimal residual disease of breast cancer. Molecular Pathology, 55, 177-181.

Dar, S. A., J. G. Kuenen \& G. Muyzer, 2005. Nested PCR-denaturing gradient gel electrophoresis approach to determine the diversity of sulfate-reducing bacteria in complex microbial communities. Applied
\& Enviromental Microbiology 71, 2325 2330.

Engelbrecht, F., C. Dickneite, R. Lampidis, M. Götz, U. DasGupta \& W. Goebel, 1998. Sequence comparison of the chromosomal regions encompassing the internalin $\mathrm{C}$ genes (inlC) of Listeria monocytogenes \& L. ivanovii. Molecular Genetics \& Genomics, 257, 186-197.

Ercolini, D., 2004. PCR-DGGE fingerprinting: Novel strategies for detection of microbes in food. Journal of Microbiological Methods, 56, 297-314.

Eruteya, O. C. \& S. A. Odunfa, 2014. Species and virulence determination of Listeria monocytogenes isolated from goat meat in Port Harcourt, Nigeria. International Journal of Current Microbiology \& Applied Sciences, 3, 32-39.

Farnleitner, A. H., N. Kreuzinger, G. G. Kavka, S. Grillenberger, J. Rath \& R. L. Mach, 2000. Comparative analysis of denaturing gradient gel electrophoresis and temporal temperature gradient gel electrophoresis in separating Escherichia coli uidA amplicons differing in single base substitutions. Letters in Applied Microbiology, 30, 427-431.

Guillet, C., O. J. Lambert, A. L., Monnier, A. Leclercq, F. Mechaï, M-F MamzerBruneel, M. K. Bielecka, M. Scortti, O. Disson, P. Berche, J. Vazquez-Boland, O. Lortholary \& M. Lecuit, 2010. Human listeriosis caused by Listeria ivanovii. Emerging Infectious Diseases, 16, 136-138.

Innis, M. A., D. H. Gelfand \& J. J. Sninsky, 1990. PCR Protocols: A Guide to Methods and Applications. Academic Press, San Diego, California, pp. 315-322.

Iordache, E. \& C. Tofan, 2008. The incidence of Listeria monocytogenes in milk. Journal of Agroalimentary Processes and Technologies, 14, 333-336.

Liao, C., Z. Y. Peng, J. B. Li, X. W. Cui, Z. H. Zhang, ZH., P. K. Malakar, W. J. Zhang, Y. J. Pan \& Y. Zhao, 2015. Simultaneous construction of PCR-DGGE-based predictive models of Listeria monocytogenes \& 
Vibrio parahaemolyticus on cooked shrimps. Letters in Applied Microbiology, 60, 210-216.

Liu, Z., C. Yuan \& S. B. Pruett, 2012. Machine learning analysis of the relationship between changes in immunological parameters and changes in resistance to Listeria monocytogenes: A new approach for risk assessment and systems immunology. Toxicological Sciences, 129, 57-73.

Luo, Q., M. Rauch, A. K. Marr, S. M. Altrock \& W. Goebel, 2004. In vitro transcription of the Listeria monocytogenes virulence genes inlC and $\mathrm{mpl}$ reveals overlapping PrfA-dependent and -independent promoters that are differentially activated by GTP, Molecular Microbiology, 52, 39-52.

Mavragani, D., C. Hamel \& V. Vujanovic, 2011. Species-specific PCR-DGGE markers to distinguish Pyrenophora species associated to cereal seeds. Fungal Biology, 115, 169-175.

McAuliffe, L., R. J. Ellis, J. R. Lawes, R. D. Ayling \& R. A. Nicholas, 2005. 16S rDNA PCR and denaturing gradient gel electrophoresis; a single generic test for detecting and differentiating Mycoplasma species. Journal of Medical Microbiology, 8, 731739.

McCammon, M. T., J. S. Gillette, D. P. Thomas, S. V. Ramaswamy, I. I. Rosas, E. A. Graviss, J. Vijg \& T. N. Quitugua, 2005. Detection by denaturing gradient gel electrophoresis of $p n c A$ mutations associated with pyrazinamide resistance in Mycobacterium tuberculosis isolates from the United States-Mexico border region. Antimicrobial Agents \& Chemotherapy, 6, 2210-2217.

McLauchlin, J., 1997. The identification of Listeria species. International Journal of Food Microbiology, 38, 77-81.

Mead, P. S., L. Slutsker, V. Diets, L. F. McCaig, J. S. Bresee, C. Shapiro, P. M. Griffin \& R. V. Tauxe, 1999. Food-related illness and death in the United States. Emerging Infectious Diseases, 5, 607-625.
Moreno, L. Z., R. Paixão, D. D. Gobbi, D. C. Raimundo, T. P. Ferreira, E. Hofer, M. H. Matte \& A. M. Moreno, 2014. Phenotypic and genotypic characterization of atypical Listeria monocytogenes and Listeria innocua isolated from swine slaughterhouses and meat markets. BioMed Research International. http://dx.doi.org/10.1155/ 2014/742032.

Muyzer, G., S. A. Hottentrager, C. Teske \& C. Wawer, 1995. Denaturing gradient gel electrophoresis of PCR-amplified $16 \mathrm{~S}$ rDNA. A new molecular approach to analyze the genetic diversity of mixed microbial communities, chapter 3.4.4, In: $\mathrm{Mo}$ lecular Microbial Ecology Manual, eds A. D. I. Akkermans, J. D. Can Elsas, \& F. J. De Bruijn, pp. 1-123.

Nübel, U., B. Engelen, A. Felske, J. Snaidr, A. Wieshuber, R. I. Amann, W. Ludwig \& H. Backhaus, 1996. Sequence heterogeneities of genes encoding 16S rRNAs in Paenibacillus polymyxa detected by temperature gradient gel electrophoresis. Journal of Bacteriology, 178, 5636-5643.

Petersen, R. F., C. S. Harrington, H. E. Kortegaard \& S. L. On, 2007. A PCR-DGGE method for detection and identification of Campylobacter, Helicobacter, Arcobacter and related Epsilobacteria and its application to saliva samples from humans and domestic pets. Journal of Applied Microbiology, 103, 2601-2615.

Rawool, D. B., S. V. S. Malik, S. B. Barbuddhe, I. Shakuntala \& R. Aurora, 2007. A multiplex PCR for detection of virulence associated genes in Listeria monocytogenes. Internet Journal of Food Safety, 9 , 56-62.

Quigley, L., O. O'Sullivan, C. Stanton, T. P. Beresford, R. R. Paul, G. F. Fitzgerald \& P. D. Cotter, 2013. The complex microbiota of raw milk. FEMS Microbiology Reviews, 37, 664-698.

Sheu, S. J., H. C. Chen, C. K. Lin, W. H. Lin, Y. C. Chiang, W. Z. Hwang, H. Y. Tsen, 2013. Development and application of tuf gene-based PCR and PCR-DGGE methods for the detection of 16 Bifidobacterium 
species. Journal of Food \& Drug Analysis, 2, 177-183.

Tchernitchko, D., J. Lamoril, H. Puy, A. M. Robreau, C. Bogard, R. Rosipal, L. Gouya, J. C. Deybach \& Y. Nordmann, 1999. Evaluation of mutation screening by heteroduplex analysis in acute intermittent porphyria: Comparison with denaturing gradient gel electrophoresis. Clinica Chimica Acta, 279, 133-143.

Van der Hout, A. H., A. M. van den Ouweland, R. B. van der Luijt, H. J. Gille, D. Bodmer, H. Brüggenwirth, I. M. Mulder, P. van der Vlies, P. Elfferich, M. T. Huisman, A. M. ten Berge, J. Kromosoeto, R. P. Jansen, P. H. van Zon, T. Vriesman, N. Arts, M. B. Lange, J. C. Oosterwijk, H. Meijers-Heijboer, M. G. Ausems, N. Hoogerbrugge, S. Verhoef, D. J. Halley, Y. J. Vos, F. Hogervorst, M. Ligtenberg \& R. M. Hofstra, 2006. A DGGE system for comprehensive mutation screening of BRCA1 and BRCA2: Application in a Dutch cancer clinic setting. Human Mutation, 27, 654-666.

Vazquez-Boland, J. A., M. Kuhn, P. Berche, T. Chakraborty, G. Dominguez-Bernal, W. Goebel, B. Gonzalez-Zorn, J. Wehlan \& J. Kreft, 2001. Listeria pathogenesis and molecular virulence determinants. Clinical Microbiology Reviews, 14, 584-640.
Wawer, C. \& G. Muyzer, 1995. Machine learning analysis of the relationship between changes in immunological parameters and changes in resistance [NiFe] hydrogenase gene fragments. Applied \& Environmental Microbiology, 61, 22032210.

Wu, Y., R. P. Stulp, P. Elfferich, J. Osinga, C. H. Buys \& R. M. Hofstra, 1999. Improved mutation detection in GC-rich DNA fragments by combined DGGE and CDGE. Nucleic Acids Research, 27, e9.

Paper received 01.08.2016; accepted for publication 25.11.2016

\section{Correspondence:}

Department of Molecular Biology and Biotechnology, Atomic Energy Commission, P.O. Box 6091, Damascus, Syria tel. 963-11-213580; fax: 963-11-6112289, e-mail: ascientific1@aec.org.sy 